\title{
Dominar el mundo privado: Casas inteligentes y razón instrumental.
}

Domination over the private world: smarthomes and instrumental reason.

DOI: 10.32870/sincronia.axxv.n79.5a21

\author{
David Francisco Nani * \\ Katholieke Universiteit Leuven (Universidad Católica de Lovaina). (BÉLGICA) \\ CE: davidfrancisconani@gmail.com / ID ORCID: 0000-0002-8684-9616
}

\begin{abstract}
* El autor es Máster en Estudios Ibéricos e Iberoamericanos por la Katholieke Universiteit Leuven de Bélgica. Es filósofo y psicólogo de formación (Universidad Nacional, Heredia, Costa Rica). Trabaja como profesor de educación privada y labora como escritor y periodista de opinión a tiempo parcial Sus áreas de interés son la filosofía de la ciencia, la filosofía social y política (especialmente los derechos humanos), y la fenomenología merleau-pontiana. E-mail: davidfrancisconani@gmail.com
\end{abstract}

Esta obra está bajo una Licencia Creative Commons Atribución-NoComercial 4.0 Internacional

Recibido: $28 / 08 / 2020$

Revisado: 03/10/2020

Aprobado: $12 / 11 / 2020$

\section{RESUMEN}

Este trabajo aborda las casas inteligentes. Las analiza mediante el concepto de razón instrumental. Señala cómo las casas inteligentes trasladan al hogar el ejercicio de la razón instrumental. Demuestra la poca importancia conferida a la subjetividad, las amenazas de conferir un inmenso poder a algunos individuos, los peligros para la vida privada del hogar y los riesgos políticos de legitimar un casi completo control de los entornos.

Palabras Clave: Casas inteligentes. Teoría crítica. Tecnología. Razón instrumental. Max Horkheimer. Modernidad.

\section{ABSTRACT}

This paper deals about smarthomes. It analyses them using the instrumental reason concept. It shows how smarthomes develop the instrumental reason at a domestic level. It demonstrates the lack of importance given to subjectivity, the threats of providing a big power to some individuals, the dangers for home private life and the political risks of legitimating an almost complete control of environments. 
Keywords: Smart homes. Critic theory. Technology. Instrumental reason. Max Horkheimer. Modernity.

\section{Introducción}

La tecnología se apropia cada vez más de los ámbitos privados de los sujetos, esto se corrobora con elementos como la vigilancia digital permanente de calles, barrios y comercios. El mundo contemporáneo se constituye tecnológicamente. De acuerdo con Mumford (1992), la invención tecnológica se ha convertido en un imperativo y elaborar nuevas maravillas carece con frecuencia de un juicio crítico.

Putnam (2002) alude a que el control racional de un tópico no pasa necesariamente por los enunciados sintéticos (que son los propios de las ciencias naturales empiristas), no es monopolio de estos, sino que los contenidos valorativos también representan materia de la razón. Entonces, si ese tema es la tecnología (en la época contemporánea estrechamente ligada con la ciencia), lo valorativo será parte del análisis racional. Porque lo tecnológico trasciende el mero método e implica otras operaciones del pensamiento, aunque algunos lo ignoren.

Más allá de lo filosófico, la reflexión sobre la tecnología tiene también importancia histórica y social. Situado con base en la experiencia del percance nuclear de Fukushima en el 2011, un investigador japonés afirma: “...scientific knowledge and technology without critical reflection are not only meaningless but also, more importantly, dangerous" [...tanto el conocimiento científico como la tecnología sin reflexión crítica son no solamente insensatas, sino que también son, lo que es más importante, peligrosas] (Shimizu, 2017: 537). ${ }^{1}$ El estudio crítico de las invenciones por tanto constituye una tarea significativa.

Debido a lo anterior, el presente ensayo parte de la preocupación por analizar críticamente un fenómeno tecnológico: la invención de las llamadas "casas inteligentes". Dicho concepto, también denominado como domótica (y derivado del “internet de las cosas"), es, a grosso modo,

\footnotetext{
${ }^{1}$ (Traducción a cargo del Mtro. Juan Enrique Escobedo Guzmán, en colaboración con el equipo editorial de la revista Sincronía.).
} 
viviendas con condiciones programables. Porque al impactar, de modo eventual, un entorno inmediato y común a casi todos los sujetos (¿Cuántos no viven en una casa, edificio o apartamento?), representa un tema impostergable.

Esto aumenta su importancia tras un somero repaso acerca de la legislación acerca de las casas inteligentes en América Latina. A diferencia de Europa (cuyas leyes no analizamos aquí), en la región el tema no ha suscitado el necesario estudio político-legal. Debido a esto, un tópico de trascendencia presenta aun vacíos considerables.

Así, navegar en el sitio web de la Biblioteca del Congreso Nacional de Chile no arroja datos de ninguna ley publicada sobre domótica, casas inteligentes o internet de las cosas. En el marco del presente artículo se consultó a congresistas chilenos vía internet, pero no hubo respuesta. Para el caso costarricense, tenemos idéntica situación respecto a la página oficial de la Asamblea Legislativa de Costa Rica, o sea la ausencia de leyes respecto a la temática. Esto se corrobora tras la consulta a Ester Méndez (Comunicación personal, 27 de abril del 2020), asesora legislativa que afirma la carencia de legislación.

Las páginas web del Senado y del Congreso de México tampoco revelan la existencia de leyes para regular las casas inteligentes. $Y$ tras consultas mediante correo a diputados no hubo respuesta. Como resulta deducible, hay vacíos en torno a un tópico delicado, vacíos que muy posiblemente se deben a la novedad del fenómeno en la escena pública. Entonces, en un contexto tal, resulta de trascendencia el aporte académico.

Postula el artículo la importancia del análisis de una tecnología aplicada a la privacidad, a los entornos más cotidianos, o sea los hogares. Porque ejercer dispositivos tecnológicos de gran alcance en ese ámbito tiene repercusiones sociales de relevancia, no susceptibles de descuido $u$ omisión investigativa. Más aun, la marcada novedad de esta propuesta supone su necesario estudio y debate. El presente artículo busca demostrar si la domótica puede devenir en aparatos de control y dominación en contra de seres humanos. Y más en específico si el cosificar y mutilar el pensamiento es parte de dicha propuesta tecnológica, conllevando desde luego repercusiones para las relaciones sociales. 
En el escrito se analizan las casas inteligentes no desde Putnam, sino con base en un autor de tradición frankfurtiana, Max Horkheimer. Se retoma su concepto de razón instrumental. Y se rescata a dicho autor entre otros motivos debido a su constante preocupación por el devenir de la razón ilustrada y sus productos, dentro de los cuales puede mencionarse la tecnología contemporánea. O sea, empleamos dicha propuesta crítica pero también referimos las adyacencias teóricas necesarias (ideas como la autonomía, la libertad, etc.).

El texto se estructura con base en esta hipótesis: Las smarthomes, en tanto ejercicio de la razón instrumental, generan la difuminación del sujeto moderno, porque diluyen su libertad y su autonomía. Con esto se busca explicar cómo la racionalidad instrumental se despliega en el invento tecnológico de las casas inteligentes.

\section{2-Metodología}

El presente artículo representa un diálogo entre los llamados "estudios sociales sobre ciencia y tecnología" y la filosofía. Sobre la primera área tenemos que Kreimer (2017) defiende su carácter interdisciplinario y habla de varias etapas históricas, con diferentes discusiones académicas. Se habla de un "tercer momento", de índole contemporánea, en el cual: "Así surgen diversos cuestionamientos a la toma de decisiones públicas, al papel de los expertos, y, más en general, hacia las relaciones entre ciencia y democracia" (Kreimer, 2017: 146). Hacemos la salvedad de que nos ocupamos de una invención tecnológica, y no del campo de la ciencia.

Entonces aquí se trata de visualizar el vínculo entre la tecnología y la política. Más en específico, buscamos establecer el concepto de casas inteligentes, apelando a una caracterización amplia del invento. Luego se procede a interpretar dicho concepto desde un marco particular.

De acuerdo con la Real Academia de la Lengua (2020) el vocablo interpretar alude a: "Explicar acciones, dichos o sucesos que pueden ser entendidos de diferentes modos" (Real Academia de la Lengua, 2020: para. 3). Efectuamos lo anterior preguntándonos acerca de si la smarthome se incardina dentro de la razón instrumental, y, por tanto, si difumina la subjetividad 
moderna. Nótese así la lectura de un tema tecnológico desde la filosofía política crítica y no desde otros posibles abordajes (fenomenología, epistemología analítica, etc.).

\section{3-Concepto de casa inteligente}

Establecemos primero la definición de rigor. Las casas inteligentes son: “...the introduction of enhanced monitoring and control functionality into homes" [...la introducción de monitoreo mejorado y control de funcionalidad dentro de los hogares] (Hargreaves, y Wilson, 2017: 1) ${ }^{2}$. Entonces el término alude a la vigilancia del entorno doméstico y a la implementación de acciones en consecuencia de los inputs obtenidos, en búsqueda de mejorar la operatividad del hogar.

De acuerdo con Liu et. al. (2016), citados por Chabot et. al. (2019), la casa domótica está equipada con tecnología inteligente, la cual se halla integrada a la residencia. Contiene sensores dentro del hogar, los cuales trasmiten datos de manera directa a los smartphones. Además de los sensores, capaces de monitorear las condiciones de la vivienda (lo cual incluye el uso del video), el dispositivo permite controlar el ambiente interno, por ejemplo, cerrando puertas o regulando la temperatura (Chabot et. al., 2019).

El soporte de las operaciones narradas lo constituye la internet, mediante un sistema computacional que consta de un computador central, así se permite las acciones anteriores y también la vigilancia (Casas Inteligentes Diseño y Construcción, 2016). De tal modo, los datos de la casa pasan a ser dominio de dicho computador. Smarthome (2016) señala la posibilidad de controlar la vivienda desde la tablet, la laptop, el smartphone y el Smart TV, también de abrir el portón de la cochera mediante un sistema GPS incorporado al automóvil.

Diferentes autores postulan los beneficios del internet de las cosas y la domótica. Chabot et. al. (2019), citando a Rantz et. al. (2015) y a Liu et. al. (2016), establecen que las casas inteligentes son utilizadas por adultos mayores con enfermedades crónicas y discapacidades, personas con

\footnotetext{
2 La obra de Hargreaves, y Wilson, (2017) se centra en las percepciones y las demandas de mercado de los usuarios, pero resulta parca en lo respectivo a la propuesta en sí de las casas inteligentes. (Traducción a cargo del Mtro. Juan Enrique Escobedo Guzmán, en colaboración con el equipo editorial de la revista Sincronía.).
} 
requerimientos de cuidado permanente. Azimi et. al. (2017) afirman la utilidad de la internet de las cosas para monitorear el adecuado uso de medicamentos, la nutrición y la seguridad de los ancianos. Marques et. al. (2019), señalan su posible empleo para un mejor control del gasto energético, y su utilidad en aras de optimizar la calidad de vida.

Aun cuando las diferentes empresas convergen en la mayoría de los aspectos propios de la casa inteligente, también hay matices que deben señalarse. De acuerdo con Keydome ${ }^{3}$ (2016) este tipo de vivienda permite saber detalles minuciosos, por ejemplo, si una llave está abierta o si una persona se cayó adentro del inmueble. Una vivienda inteligente, según Casas Inteligentes Diseño y Construcción (2016), permite una interacción a distancia de posibilidades ilimitadas del dueño con respecto a su residencia, hace posible abrir y cerrar ventanas, portones eléctricos y puertas, apagar y encender luces y aparatos, encender la calefacción, ver y escuchar lo que acontece en la casa.

Chabot et. al. (2019) establecen limitaciones de las casas inteligentes. Al respecto afirman que pueden crear dependencia para los adultos mayores, e incurrir en brechas entre las capacidades de estas personas y las exigencias del paquete tecnológico (Chabot et. al., 2019). También señalan la necesidad del consentimiento del usuario acerca de la recolección de sus datos privados, y de garantizar la privacidad de dicha información (Chabot et. al., 2019).

Esto último es descrito como una vulnerabilidad del sistema. Marques et. al. (2019) analizan los dispositivos del internet de las cosas propio del sector salud. Aducen que, aun cuando se han ideado programas mejorados, la privacidad y la seguridad siguen siendo desafíos para dichos sistemas (Marques et. al., 2019). Lo anterior se debe al manejo conectado de los datos, justamente: "Network communications are exposed against network security attacks because of the broadcast nature of the transmission medium" [Las redes de Comunicaciones son expuestas en contra de los ataques de las redes de seguridad debido a la naturaleza de la emisión del medio de transmisión]

\footnotetext{
${ }^{3}$ Varias de las empresas citadas laboran en países latinoamericanos. Entonces, las casas inteligentes ya son parte de la realidad de América Latina, responden a las características de sus formaciones sociales, particularmente a la necesidad percibida de seguridad.
} 
(Marques et. al., 2019: 18). ${ }^{4}$ El asunto adquiere un carácter perentorio dentro de la argumentación. Así, Marques et. al. (2019) instan a aprobar leyes para proteger la privacidad en el marco de las mencionadas tecnologías.

Smarthome (2016) señala el concepto de domótica, este consiste en la aplicación de la tecnología en un hogar para controlar y automatizar sus sistemas, aportando a su gestión con acciones como el ahorro de energía, confort y seguridad, mediante una comunicación eficiente entre el usuario y el dispositivo. El empleo de la inteligencia artificial cambiaría el sistema:

In contrast with usual automation solutions, such intelligent technologies would allow constant monitoring of the environment and its residents and learning from their behavior. Then this knowledge can be used to update environment control rules automatically, and even predict the future changes and events, providing services for the users without intervention (or with very minimal) from the latter.

[En contraste a las soluciones automatizadas usuales, tecnologías tan inteligentes permitirían el monitoreo constante del medio ambiente y sus residentes, y el aprendizaje de su comportamiento. Entonces, este conocimiento puede ser usado para actualizar automáticamente las reglas de control del ambiente, e incluso predecir eventos y cambios futuros, proveyendo servicios para los usuarios sin la intervención (o con una intervención mínima) de los éstos] (Maskeliūnas, Damaševičius, y Segal, 2019: 11). ${ }^{5}$

Así vemos como las condiciones de control del entorno y de las personas pueden estrecharse mucho más que en la actualidad y alcanzar niveles casi de perfección. Porque una vez más vemos el binomio de conocer el espacio hogareño, anticipar sus fenómenos y manipularlo con gran detalle.

De acuerdo con D-Link (2016) el sistema de vigilancia cuenta con cámaras para monitorear el ingreso de cualquiera. El sistema descrito por Casas Inteligentes Diseño y Construcción (2016) incluye una conexión con el microondas, así, en caso de rotura intencional de los cables de energía y

\footnotetext{
${ }^{4}$ (Traducción a cargo del Mtro. Juan Enrique Escobedo Guzmán, en colaboración con el equipo editorial de la revista Sincronía.).

5 (Traducción a cargo del Mtro. Juan Enrique Escobedo Guzmán, en colaboración con el equipo editorial de la revista Sincronía.).
} 
teléfono, dicho ligamen permite la comunicación con una empresa de seguridad, la policía y el propietario del inmueble.

La empresa Keydome (2016) por su parte ofrece un sistema de seguridad "unificado", controla sensores, cámaras de seguridad, y un método de alarmas capaces de vigilar el hogar por internet o a nivel local. También es posible contar con un procedimiento de automatización, integrable al de seguridad, de ese modo si un sujeto se traslada a un dormitorio, una respuesta coordinada lo detecta y enciende las luces, o bien, si alguien ingresa a la alcoba, se emite una alerta al celular del interesado.

Para maximizar las ventajas, GSMA y KRC Research (2016) proponen que los diferentes servicios y dispositivos deben tener la disposición de interactuar entre sí, dándose una interoperatividad y una colaboración entre compañías de diferentes sectores, pues además de las casas inteligentes se habla del "internet de las cosas", el comando a distancia de los distintos aparatos. Habiéndose descrito el sistema de casas inteligentes, pasamos a esbozar la idea de razón instrumental.

\section{4-Concepto de razón instrumental en Max Horkheimer}

¿Qué era la razón antes de la sociedad industrial? Horkheimer (1969) nos habla de que durante siglos predominó una visión distinta. Así, afirma:

Tal visión afirmaba la existencia de la razón como fuerza contenida no sólo en la conciencia individual, sino también en el mundo objetivo: en las relaciones entre los hombres y entre clases sociales, en instituciones sociales, en la naturaleza y sus manifestaciones. Grandes sistemas filosóficos, tales como los de Platón y Aristóteles, la escolástica y el idealismo alemán, se basaban sobre una teoría objetiva de la razón. Ésta aspiraba a desarrollar un sistema vasto o una jerarquía de todo lo que es, incluido el hombre y sus fines. El grado de racionalidad de la vida de un hombre podía determinarse conforme a su armonía con esa totalidad (Horkheimer, 1969: 16). 
Vemos aquí una racionalidad según la cual pueden pensarse los diferentes ámbitos de la existencia humana, así también sus relaciones, y más aún, los propósitos de las acciones. Esto último resultaba de gran importancia, sobrepasaba la evaluación de los medios; el ejercicio de la razón conducía, en su nivel más alto, a concertar el orden objetivo de lo racional, según lo entendía la filosofía, con la existencia del ser humano, ubicando en esto la autoconservación y el interés (Horkheimer, 1969). Entonces deducimos una razón integradora y no taxativa a lo subjetivo. La razón subjetiva, en contraste con lo anterior, refiere de manera exclusiva al vínculo guardado entre un objeto o concepto con un fin, y no al propio concepto u objeto (Horkheimer, 1969). Así los pensamientos o las cosas sirven para algo más, desterrándose la idea de metas racionales en sí y la discusión de la superioridad de una meta respecto a otras con arreglo a la razón (Horkheimer, 1969). La razón subjetiva se conoce como razón instrumental y sobre tal concepto los desarrollos teóricos son $\operatorname{vastos}^{6}$

En la obra Crítica a la razón instrumental se plantea:

La razón aparece totalmente sujeta al proceso social. Su valor operativo, el papel que desempeña en el dominio sobre los hombres y la naturaleza, ha sido convertido en criterio exclusivo. Las nociones se redujeron a síntesis de síntomas comunes a varios ejemplares (Horkheimer, 1969, p. 32).

Opera entonces una hiper-simplificación de la razón, un pensar mutilado. Representa las tendencias actuales de la sociedad. Constituye una razón preocupada más por el cómo que por el qué, reducida a mero procedimiento (Nateras, 2009). Según Germain (2019) la racionalidad científica moderna es cultivada bajo el propósito de su uso potencial. Horkheimer (2000) señala que la ciencia, al anticipar los fenómenos de la realidad mediante la ley causal natural, es capaz de pasar al control del entorno y también de los contextos sociales, controlar el mundo parece ser el derrotero. En otra obra se caracteriza el término de razón instrumental:

\footnotetext{
${ }^{6}$ La razón instrumental constituye el concepto a utilizarse en el escrito. Para efectos aclaratorios, debemos decir que la razón objetiva no es idealizada por Horkheimer (1969) y al respecto establece críticas. Sin embargo, escapa a los fines del presente artículo un análisis detallado de la razón objetiva.
} 
Todo uso que va más allá de la sintetización técnica de datos fácticos, que sirve de ayuda, se ve extirpado como una huella última de la superstición. Las nociones se han convertido en medios racionalizados, que no ofrecen resistencia, que ahorran trabajo. Es como si el pensar mismo se hubiese reducido al nivel de los procesos industriales sometiéndose a un plan exacto; dicho brevemente, como si se hubiese convertido en un componente fijo de la producción (Horkheimer, 1969: 32).

Así, la razón se reduce a un conjunto de operaciones tendientes a lograr objetivos, tiene por escenario taxativo un mundo hiper-empírico, todo pensar distinto de la eficiencia de ese sistema de procedimientos resulta ajeno a la razón instrumental, en cuenta la pregunta del para qué, pues únicamente importan las operaciones. Slijepčević (2019), tras retomar a Horkheimer y Adorno (2002), aduce que en esta forma de pensar la razón se vuelve irracional. Converge Fuchs (2019) con lo anterior cuando afirma que dicha racionalidad rebaja la conciencia humana a las calidades de la máquina. Entonces deducimos el socavamiento de la razón mediante un ejercicio de ésta que la cercena y empobrece en sumo grado, dejando vigente sólo aquellos puntuales aspectos de índole procedimental.

No en vano indica Horkheimer (2000) que la sociedad industrial crea individuos permanentemente alertas, orientados a lo inmediatamente práctico, dispuestos siempre y en todas partes con la misma vigilancia. La obra Teoría tradicional y teoría crítica, en su anexo denominado Razón y autoconservación, también señala que esta forma de razonar disocia lo operativo de lo valorativo, la razón y la ciencia no tienen vínculo con el juicio de valor (Horkheimer, 2000). El quehacer científico no piensa rumbos, sino sólo procedimientos correctos para lograr fines. Acerca de tal fenómeno se tiene: "La ciencia carece de autorreflexión para conocer los motivos sociales que la impulsan hacia un lado, por ejemplo, hacia la Luna, y no hacia el bien de la humanidad" (Horkheimer, 1976: 56-57). Así, si todo se restringe a los avatares del procedimiento, no podemos reflexionar sobre fines. 
Aquí puede notarse la crítica hacia un quehacer científico incapaz de pensar los fines de su actividad, y dada esa falencia, resulta presa de las corrientes imperantes en la sociedad industrial. En consecuencia, el mundo producido por esta razón pragmática es uno donde todo sirve para algo y debe ser útil para ser reconocido (Galafassi, 2004). De acuerdo con Zhang (2013) la Revolución Industrial priorizó el instrumento por sobre el fin, preconizó la eficiencia, la precisión y la objetividad; pero la riqueza de los significados del mundo de la vida disminuyó.

Al respecto puede señalarse la lectura de López (2000), dicho autor argumenta el evitar el juicio sobre los valores y los fines de la acción como signo de esta racionalidad. También le es característico el centrarse en la pura gestión de medios, limitando la capacidad para aprehender y determinar el sentido de las acciones, rasgo de la conciencia (López, 2000). La razón instrumental se relaciona con el rebajamiento de la ética en tanto labor, pues las investigaciones basadas en dicha perspectiva soslayan los aspectos morales porque miran con indiferencia los propósitos (Shimizu, 2017). Aplicando un término de Marcuse (1991), podemos ver cómo se torna unidimensional el pensar humano.

La consulta de la obra de Hinkelammert (2003) aclara también la idea, la razón instrumental se preocupa únicamente por la eficiencia y la eficacia del cálculo medio-fin, las consecuencias del fin, una vez realizado, para la naturaleza y para la sociedad no importan a este tipo de pensamiento. Y según Slijepčević (2019), quien comenta a Horkheimer y Adorno (2002), la visión científica del mundo, que domina occidente, entiende la naturaleza de forma mecánica, y esto repercute en la destrucción del medio.

Por añadidura, algunos estudiosos del pensamiento frankfurtiano indican la trasmutación de los medios en fines para el caso de la sociedad industrial (Galafassi, 2004); además señalan que los sujetos no se preocupan por comprender la finalidad (Nateras, 2009). Por su parte Zhang (2013) advierte que la expansión de esta racionalidad puede desarrollar fuerzas opresivas y conducir a la crisis de la propia modernidad. Según De Genaro (2017) en dicho tipo de razón los sujetos se transforman en engranajes sin comprender ni dominar el proceso, al cual sin embargo se adaptan 
bajo el supuesto infundado de estarse imponiendo en éste. Lo descrito se asemeja al concepto marxista de falsa conciencia y tiene efectos psicosociales de amplio impacto.

El mundo instrumental ejerce efectos en las colectividades humanas. Los sujetos se amoldan a responder a señales, a necesitar directivas y mostrarse obedientes porque su necesidad de órdenes crece; todo esto decanta en una pérdida progresiva de las reacciones espontáneas (Horkheimer, 1970). Porque si los fines ya no importan y preocupa solo el ser operativo, el producto lo constituye un sujeto dúctil, incapaz de determinar ni el significado ni el propósito de sus acciones, menos aún de proponer caminos. A fin de cuentas, la subjetividad humana resulta amenazada por la imposición de fuerzas sociales impersonales, dichas fuerzas nos reconceptualizan según los dictados de los aparatos técnicos de control (Germain, 2019). Así podemos afirmar la existencia de procesos de deshumanización en la sociedad industrial contemporánea, los cuales denotan el tipo de individuo necesario a sus fines: el funcionario sumiso y eficiente, no el ser creativo ni crítico.

Debido a lo anterior, la máquina industrial se asemeja a su creador, los seres humanos se adaptan a problemas propuestos, pero cada cual se torna más solitario, dado que la máquina puede trabajar y calcular, pero no identificarse con otros ni tener ocurrencias (Horkheimer, 1970). Todo lo anterior trae consigo un sujeto predecible, moldeable, incapaz de afirmarse en las relaciones sociales. Este proceso muestra una impronta sociopolítica profunda:

Pese a toda su actividad, los hombres se tornan más pasivos; pese a todo su dominio de la naturaleza, se vuelven más impotentes frente a la sociedad y a sí mismos. La socie dad por sí misma trabaja con miras al estado atomizado de masas, deseable para los dictadores (Horkheimer, 1970: 30).

Entonces tenemos que la sociedad industrial genera seres humanos instrumentales, y de su adaptación al mundo administrado al seguimiento dócil de una dictadura no hay mucha distancia. Estos seres humanos, bajo tal yugo, no son autónomos. Aquí resulta evidente aquel principio de la Escuela de Frankfurt según el cual la modernidad produce los mecanismos responsables de su propia aniquilación. Así, respecto al diagnóstico de estos pensadores, tenemos: "They maintained 
that the modern project is driven by the imperative to realise ever more effective means of controlling the physical and social environments" [Ellos sostuvieron que el proyecto moderno es motivado por el imperativo de lanzar continuamente medios más efectivos de control de los contextos físicos y sociales] (Germain, 2019: 128). ${ }^{7}$ La promesa de autonomía se haya extinta en un universo social como el aquí descrito, donde reina la dominación y no la soberanía de los sujetos.

Y esto atenta contra los pilares de la propuesta moderna. Así para Locke (2005) los hombres tienen la posibilidad de disponer de sus vidas y sus bienes según les parezca mejor, en ello radica la libertad, caso contrario acontece la esclavitud. Pero un aparato social como el descrito socava esa libre iniciativa respecto a las existencias de los individuos, porque su proceso formativo no concede que los hombres planeen sus vidas ni les den una dirección.

También porque en Kant (1963) los sujetos se dan a sí mismos la ley moral universal merced a la razón y no a los influjos sensibles, cuando acontece lo último carecemos de sujeto autónomo. Contextualizando la autonomía moderna a nuestros días, resulta válido citar a Hortal (2002), para quien ese concepto alude a la capacidad y la prerrogativa de cada sujeto para proyectar su vida de acuerdo con sus deseos, ideales e intereses propios, sin intromisiones.

El pensamiento de la Escuela de Frankfurt, en su uso crítico de Kant, aplica el difuminado de la autonomía como fruto de la sumisión a los poderes del mundo. Estos pueden ser ya estados explícitamente autoritarios (independientemente de su signo ideológico), o ya los más sutiles mecanismos de la sociedad industrial capitalista tardía (sociedad de masas, confort material sin libertad, industria de la cultura, etc).

Ante un panorama con las calidades antedichas, la teoría crítica tiene la tarea de expresar lo generalmente inexpresado, de señalar los costos del progreso, los factores que, como fruto del proceso, destruyen inclusive el concepto de sujeto autónomo (Horkheimer, 1976). La perspectiva implica un arduo reto para los tiempos venideros:

\footnotetext{
${ }^{7}$ (Traducción a cargo del Mtro. Juan Enrique Escobedo Guzmán, en colaboración con el equipo editorial de la revista Sincronía.).
} 
Al final, si alguna catástrofe no destruye la vida por completo, habrá una sociedad totalmente administrada, automatizada, que funcionará de un modo estupendo, en la que el individuo puede ciertamente vivir sin preocupaciones materiales, pero ya carece de toda importancia (Horkheimer, 1976: 65).

Tenemos aquí el tópico de la difuminación del sujeto moderno, entendido como particularidad. De acuerdo con Fuchs (2019) los intentos de cosificar la consciencia traen consigo la manipulación de los seres humanos. Según Fromm (2002) es un rasgo de la sociedad totalitaria que el individuo resulta desprovisto de su valor. Como ladrillos de una construcción, los seres humanos sólo se amoldan, cercenando sus potencialidades humanas.

De acuerdo con López (2000) constituye un elemento básico de la Escuela de Frankfurt el tema de pensar la totalidad. Aquí se adiciona que dicho criterio sí permite el análisis de los contextos, los propósitos (presentes y potenciales) y las consecuencias (contemporáneas y posibles) de los proyectos modernos. No en vano temas como estos representan aspectos cardinales del freudomarxismo ¿Es síntoma la domótica de la racionalidad aquí descrita? Dicha pregunta se responde en el siguiente apartado.

¿Hay alguna salida a estos desafíos? En su obra Crítica a la razón instrumental, Horkheimer (1969) postula algunas posibles soluciones, entre estas que la razón busque la verdad y no el dominio, así como la conciliación con la naturaleza. En otro libro, dicho autor invita a una racionalidad abarcadora e integral que vaya mucho más allá del mero procedimiento: "Para ser verdadera, la ciencia debería conducirse críticamente para consigo misma y para con la sociedad que ella produce" (Horkheimer, 1976: 57). En ese sentido, invita Horkheimer (1976) a pensar los motivos que impulsan una u otra línea de investigación, porque según dicho analista la teoría crítica surgió de las ideas relativas a un mundo mejor, dirigiendo su trabajo frente a la sociedad y frente a la ciencia. Vemos por tanto implícito el tema de la totalidad.

También se habla del rescate de la autonomía de los individuos, de tal manera que se conserve para el mayor número posible de seres humanos la autonomía del sujeto, siendo así 
posible el desarrollo de sus fuerzas (Horkheimer, 1976). De manera patente se expone: "Nosotros queremos conservar el mayor tiempo posible la independencia interna y la satisfacción interna del individuo humano, y con ello la oportunidad de su solidaridad con los otros seres humanos" (Horkheimer, 1976: 66). Entonces deducimos la teoría crítica como una propuesta en pro del rescate de la subjetividad frente al aparataje político y social.

\section{5-Las casas inteligentes como razón instrumental}

Las casas inteligentes suponen un control casi total del entorno privado. Este es posibilitado por los avances tecnológicos. Al respecto pueden formularse distintas reflexiones. Primero debemos decir que si bien el sistema provee comodidades (atención cercana a personas mayores, ahorro de energía, entre otras), el "precio a pagar" resulta sumamente alto, equivale al menoscabo de la privacidad, con las concomitancias respectivas.

Y una privacidad menoscabada, así como individuos manipulables por la anticipación de sus actos, son factores que atentan incluso contra las libertades individuales. Que la internet cuente con acceso a las vidas privadas permite desde ya la dilucidación de tendencias de personalidad, gustos, etcétera, en un proceso en el cual el usuario no asiste al mercado como sujeto que delibera con libre arbitrio, sino bajo manipulación al conocérsele de antemano y encausársele mediante mecanismos y supuestos que él desconoce. Porque con dicha información muchas empresas pueden imponerle al sujeto lo que quieren venderle, de manera que éste no compre el bien deseado o requerido ni siga sus intereses propios, sino los de otros. Dado el estrecho conocimiento de la personalidad, por medio de mecanismos tecnológicos como el aquí descrito (y otros más), tal proceso sería posible de lograr.

Y, más aun, recordemos que, de incorporarse la inteligencia artificial a dichos sistemas, la anticipación del sujeto humano será casi total, desde luego dicha propuesta será el óbito de ideas como la autonomía y la libertad. Porque aplicar dichas creaciones favorecerá de gran forma el perfeccionamiento de las dominaciones descritas. 
Así podemos argumentar una crítica en clave moderna. Para el pensamiento de John Locke (2005) la libertad individual viene a ser el sumo bien, quien la pierde queda a merced de su dominador, éste puede disponer a su antojo de la vida y los bienes de aquel individuo. Siguiendo tal razonamiento, podemos establecer una jerarquía en la cual las libertades individuales están primero, incluso respecto a la salud y el confort. Sin embargo, las casas inteligentes implican la renuncia a lo primero en pos de lo segundo. Esto puede conducir al despotismo.

En términos de Horkheimer, esa renuncia a las libertades a cambio de bienes materiales representa un signo clásico de la sociedad industrial. Los sujetos dominan la naturaleza (referida aquí al entorno doméstico), incluso en el marco de su propio organismo (conquista de una mejor salud), pero pierden el control sobre sus vidas. Porque sus existencias pasan a estar integralmente monitoreadas e influidas por terceros, amén de todo lo que ello puede traer como consecuencia. Todo esto aparece como fruto de la racionalidad instrumental, en su negación del sujeto humano.

Segundamente se establece la domótica y el internet de las cosas en tanto casos de un tipo de racionalidad destinada al dominio del mundo y sus objetos, ese afán se inscribe en la modernidad. Aquí el interés reside en el estrecho control del ámbito privado. En efecto, esas ansias modernas por controlarlo todo parecen alcanzar un nuevo episodio con las casas inteligentes y el internet de las cosas.

La dominación de la naturaleza y de los entornos sociales alcanza un rango mayor. Ahora personas puntuales, individuos si se quiere, pueden apropiarse de esta racionalidad instrumental y aplicarla en su vida cotidiana. Por tanto, estamos en presencia de un nuevo peldaño de la imposición de la racionalidad moderna, el usuario tiene la capacidad de convertirse en un pequeño "Gran Hermano" 8 de su propio hogar. Así, un proceso macro, como lo es el ejercicio de la razón instrumental en las sociedades, tiene ahora un alcance hogareño y coloca en una posición de poder nunca antes vista a determinados sujetos (quienes controlan el sistema desde los espacios externos a la casa).

8 Término de la novela 1984, del inglés George Orwell. 
Más aún, la idea de una estrecha vigilancia y una constante manipulación del entorno, decantarían en la naturalización de una racionalidad: es válido el control pleno del espacio. Entonces, esto constituye una idea extrapolable a nivel político, porque si un ciudadano, en aras de la pretendida salvaguarda, practica este concepto, puede pensarse que un Estado actuará de idéntica forma. También para cumplir con pretendidas potestades e intereses comunes, el aparato estatal puede poner en práctica una tecnología de acceso irrestricto y de estrecha incumbencia en la cotidianidad. Esta proyección de tener no sólo casas inteligentes sino también "ciudades inteligentes" ya es visualizada por los teóricos del internet de las cosas: "As mentioned above, the concept of cognitive technologies for smart environments goes far beyond the home environment and covers smart cities and public loT services" [Como se mencionó anteriormente, el concepto de tecnologías cognitivas para entornos inteligentes va mucho más allá del contexto doméstico y envuelve ciudades y servicios loT (el internet de las cosas) inteligentes] (Maskeliūnas, Damaševičius, y Segal 2019: 7). ${ }^{9}$ Esto arroja posibilidades de un control completo tanto de viviendas como de urbes enteras.

Pero una acción de tal guisa implica la saturación de la sociedad civil a cargo del Estado, práctica contraria a ciertos preceptos modernos, por ejemplo, la autonomía y libertad de los sujetos. Una vez más, la misma modernidad realiza el óbito de sus propias premisas y promesas. Con las casas inteligentes resulta ostensible un ejemplo del moderno e indesligable dúo de conocer y manipular, el conocimiento como fuente de poder y control, en este caso de la residencia, pero con posibles extensiones y efectos aún más problemáticos. Lo anterior da paso a pensar otros temas.

Esta tendencia a la dominación del hogar se inscribe como un ejemplo típico de instrumentalización de la razón, solo importa la eficacia de los bienes tecnológicos para lograr el cometido del control. Porque en ningún momento se valora la posibilidad de que el propietario puede no ser un sujeto idóneo para tener una cuota tan alta de poder, siendo necesaria una

\footnotetext{
${ }^{9}$ (Traducción a cargo del Mtro. Juan Enrique Escobedo Guzmán, en colaboración con el equipo editorial de la revista Sincronía.).
} 
evaluación previa de esta persona; priva lo procedimental, y desde luego, lo comercial. El proyecto de la domótica no se interesa por dichos tópicos, únicamente le importa la eficiencia y satisfacer las demandas del posible comprador: seguridad, ahorro de energía y manipulación del entorno privado. Salvo muy marcadas excepciones, la bibliografía que se consultó revela este tipo de ejercicio.

Un asunto de gran relevancia crítica se refiere a las posibilidades de dominación de otros seres humanos, pues el propietario tiene la potestad del sistema de la casa inteligente. Con estos dispositivos cuenta con amplísimas posibilidades para vigilar a otras personas, por ejemplo, sus hijos, con o sin su consentimiento o conocimiento. También puede ejercer un control estrecho respecto a sus acciones, por ejemplo, no permitiéndoles el egreso o el ingreso al inmueble, apagando o prendiendo artefactos, etc.

De tal modo, los dispositivos mencionados pueden dar paso a la comisión de dominaciones. Medios capaces de garantizar la seguridad del hogar de cara al mundo externo, bien pueden traer consigo la inseguridad de cara al mundo interno de la casa. Piénsese en una familia donde el padre golpea a sus hijos, o la nieta agrede a la abuela de forma psicológica ${ }^{10}$. Pero las consecuencias de la smarthome para los pobladores de la vivienda, una vez consolidado el sistema, no importan desde este tipo de racionalidad.

El aspecto de brindar datos del hogar a un computador central, aunado a lo establecido acerca de la colaboración entre distintas empresas del sector, tienen por efecto el acceso irrestricto de otros al entorno privado, esto les permite conocer a los sujetos residentes en esas casas, dilucidar patrones de interacción cotidiana e incluso anticipar sus conductas. Las promesas de confidencialidad se relativizan. Primero porque se habla de la cooperación entre distintas empresas del ramo, las cuales compartirían información (lo privado se hace público).

Además, porque únicamente los servicios de una empresa, entre todas las analizadas aquí, señalan tal cláusula de confidencia, en las otras ese precepto no se menciona. Y, en tercer lugar,

\footnotetext{
${ }^{10}$ Este hecho no debe descuidarse, particularmente en un contexto como el de América Latina, donde la violencia intrafamiliar tiene cifras muy grandes.
} 
porque los derechos a la confidencialidad han sido vulnerados en los últimos años, y aquí está en juego no una llamada telefónica o un correo electrónico, sino datos muchísimo más abundantes y explotables con múltiples propósitos.

Lo anterior decanta en la posibilidad de control por parte de las empresas vigilantes con respecto a las vidas de las personas, háblese de temas de consumo y publicidad, con tan fidedigna información se abre el portillo para prever costumbres de compra, proporcionándose luego al mercado datos de primerísima mano, este a su vez podrá tomar la información para depurar su oferta e incidir de modo más exitoso. Un ejercicio así atenta contra la autonomía del consumidor, pues permite su manipulación. Otra posibilidad de control, relacionada también con el acceso a los patrones cotidianos de interacción, consiste en poder analizar las dinámicas psicosociales de los habitantes de cada casa, por no hablar de la capacidad para acceder a las afinidades políticas de poblaciones enteras.

¿Por qué vulnerar el mundo privado atenta contra la libertad y la autonomía? Porque recordemos que el proceso funciona en binomio, se adquieren datos del entorno personal y se procede en consecuencia. Son previstas las conductas y se actúa de manera acorde. Dicho proceso ahoga la espontaneidad, que al menos para la Escuela de Frankfurt es un rasgo del sujeto autónomo. También porque el aparato tecnológico tendería a inundar de estímulos a los sujetos, estos pasarían a ser meros receptores pasivos de comodidades, sujetos que "solo responden a señales", parafraseando a Horkheimer (1970). Resulta lícito decir dicho argumento dadas las obvias tendencias del mundo tecnológico, que refinan sus mecanismos.

Además, si la autonomía radica en constituir nuestras vidas según nos parezca mejor, necesitamos hacerlo teniendo al menos un entorno donde reflexionar libremente al respecto, ese escenario lo constituye la vida privada. Por su parte Peña (2001) aduce que suele plantearse la autonomía como una de las dimensiones de la privacidad. Pero aquí planteamos más bien la privacidad como uno de los garantes de la autonomía. Si se atenta contra el mundo privado, se desvirtúan los sujetos autónomos. 
Para ejercer la autonomía necesitamos pensar, lo cual supone las condiciones necesarias en aras del ejercicio de la razón. De acuerdo con Bernal (2014) para ser autónomos debemos poder elegir entre distintas opciones, y esas opciones deben ser informadas y libres de coerción y de influencias excesivas o indebidas. Así, si el escenario donde pensamos como dirigir nuestras vidas resulta objeto de manipulaciones, ergo se difumina la autonomía. A esto súmese el posible dominio de individuos, por ejemplo, en una familia violenta.

El tópico de los posibles usos de la información cobra mayor gravedad si se recuerda la herramienta básica del sistema: internet, un medio sumamente permeable donde la protección y los cercos a los flujos de datos se vulneran, por ejemplo, debido a hackers. Así podemos recordar que la tecnología wireless muestra deficiencias en el tema de la seguridad: "In spite of the soaring interest for the analysis and exploitation of pervasive communities in the wireless domain, in regard to privacy very little has been achieved" [A pesar del creciente interés por el análisis y explotación de las comunidades presentes en el dominio inalámbrico, muy poco se ha logrado en relación a la privacidad] (Bilogrevic et. al. 2012: 371). ${ }^{11}$ A pesar del avance en otras áreas, el resguardo de los registros sigue siendo débil.

Más aun, en el campo del presente escrito el problema se corrobora de manera patente. De acuerdo con Vargas (7 de julio de 2016), el llamado "internet de las cosas" (IOT por sus siglas en inglés) ha recibido críticas dadas sus carencias en materia de seguridad. La empresa HewlettPackard realizó un estudio en el 2015, el análisis examinó diez dispositivos de loT, entre estos televisores, tomas de corriente, alarmas, cerraduras de puertas y cámaras web; los hallazgos revelaron que el $90 \%$ de los artefactos recolectaba información personal de sus usuarios, el $70 \%$ se conectaba a servicios no encriptados de internet que podían ser fácilmente accesibles a cibercriminales, y el $80 \%$ mostró deficiencias de privacidad (Vargas, 7 de julio de 2016). Nada conectado a internet es totalmente seguro (Naughton, 6 de diciembre de 2015).

\footnotetext{
11 (Traducción a cargo del Mtro. Juan Enrique Escobedo Guzmán, en colaboración con el equipo editorial de la revista Sincronía.).
} 
El internet de las cosas guarda un estrecho ligamen con las casas inteligentes, el segundo dispositivo puede incluirse en el primero, ambos emplean la conexión a la web como herramienta fundamental. GSMA y KRC Research (2016) hermanan ambos tipos de servicio. Entonces, la situación expuesta transformaría, aún más, el ámbito privado en ámbito público, accesible a terceros, aún sin el consentimiento de los sujetos.

Pero ¿Habrá de veras apetencia de datos de personas y familias en el mundo contemporáneo? Por supuesto que sí. Al menos desde el campo de la política contamos con evidentes pruebas. En primer lugar, la Patriot Act de la Administración Bush. Y en el contexto latinoamericano, recuérdese que Venezuela, Cuba y Nicaragua se hallan bajo regímenes dictatoriales.

El caso de un país otrora reputado de respetuoso de los derechos humanos funge de cuarto ejemplo. Así en Costa Rica el Poder Ejecutivo, a contrapelo de la ley y de la institucionalidad, recabó información privada de los ciudadanos sin su consentimiento y sin orden de un juez, al emitir una ley según la cual: “...también se brindará acceso a la UPAD a información de carácter confidencial con la que cuenten las instituciones públicas cuando así se requiera" (№ 41996-MP-MIDEPLAN, 2019). Estos hechos generaron incluso allanamientos y demandas penales contra el Presidente (Ruiz, 29 de Febrero de 2020).

Un invento como la domótica, al ser voluntario, otorga amplias potestades a los estados y legitima el espionaje. Con la venia de los propios sujetos, el espionaje al cual serán sometidos se naturaliza, al tiempo de que denunciar el hecho perderá relevancia. Vemos como la razón instrumental allana el camino a los ejercicios autoritarios. El tema de la renuncia a pensar los contextos, los propósitos y las repercusiones de la tecnología facilita el proceso.

Las smarthomes contribuyen con la difuminación del sujeto moderno, diluyen la autonomía y la libertad. El mundo privado se anula por el aparato tecnológico, y con esto los principios dichos no son viables. Corroboramos con el caso de las casas inteligentes que la razón instrumental genera sociedades dúctiles, acoplables a proyectos políticos de un estrecho dominio sobre los sujetos. 


\section{6-Conclusiones}

Primeramente, debemos referir la riqueza argumentativa que puede desarrollarse en el análisis. En efecto, el estudio de los temas tecnológicos desde ingresos críticos ofrece amplias vetas de desarrollo teórico ${ }^{12}$. Debido a lo anterior, abogamos por continuar este trabajo reflexivo.

Porque ni la ciencia ni la tecnología, por sí mismas, ni habrán de depararnos un mundo mejor (como prometía Comte), ni resuelven debates sobre lo deseable y lo detestable de las prácticas humanas, eso constituye tarea de los sujetos humanos. Tampoco impiden postular la existencia de umbrales que, de sobrepasarse, conecten con "mundos peores"13. Demostrar las posibilidades de dominación mediante los bienes tecnológicos tiene así gran relevancia ${ }^{14}$. Porque si somos críticos podemos antever y prevenir los riesgos y los posibles usos perversos de la ciencia y la tecnología ${ }^{15}$. Caso contrario, la apología ciega de dichas áreas del saber tiene el potencial de coadyuvar nuevos totalitarismos ${ }^{16}$.

Así, dados los resultados del presente escrito, debemos decir que la domótica encierra potenciales peligros. Es más "lo malo" que "lo bueno". Un elemento cardinal lo constituye la invasión del mundo privado al conectar al hogar con la internet. Una casa bajo monitoreo y dominio constantes representa un espacio donde toda información confidencial deja de ser privada. Esto a su vez atenta contra la autonomía, valor moderno.

\footnotetext{
12 Piénsese en el estudio de propuestas como el transhumanismo, o bien, los desarrollos de la inteligencia artificial o la biotecnología.

${ }^{13}$ La experimentación genética con embriones y la clonación de seres humanos representan una posibilidad científicotecnológica que, al menos para el mundo científico en su gran mayoría, puede (pero no debe) desarrollarse.

${ }^{14} \mathrm{Al}$ respecto tenemos: "Left unexamined, the technological drive to integrate reality will continue to render humanity redundant, or otherwise challenge our humanity as never before" [Dejada sin examinar, la motivación tecnológica para integrar la realidad continuará haciendo a la humanidad redundante, o si no retará a nuestra humanidad como nunca antes] (Germain, 2019: 133) (Traducción a cargo del Mtro. Juan Enrique Escobedo Guzmán, en colaboración con el equipo editorial de la revista Sincronía.). De tal guisa, a la pertinencia de debatir sobre las producciones científicas y tecnológicas por ser de interés filosófico, se une la relevancia social e histórica de dicho ejercicio, dadas las potenciales repercusiones de esa discusión.

15 El mundo de la ciencia ficción ha advertido, desde siglos pasados, los peligros de una ciencia y una tecnología barbarizadas. Una obra reciente es la película Ex - Machina, de McDonald y Reich (2015).

16 Esto a su vez da paso a otras preguntas, relativas a la historia de las ideas ¿Hubo critica filosófica de la ciencia y la tecnología en las épocas anteriores a los totalitarismos del siglo XX? Si existió, ¿Qué planteaban esos cuestionamientos?
} 
Las casas inteligentes se enmarcan dentro de un tipo de racionalidad cuyo principio reside en el dominio del mundo. Implican un despliegue de dicha racionalidad al espacio hogareño, instituirse en "Gran Hermano" es ahora posible dentro del ámbito doméstico. Dicho con otras palabras, la domótica constituye un capítulo más de la razón instrumental.

Esto a su vez contiene un problema derivado, consiste en la racionalización de un estrecho control social de los espacios, dándose por ejemplo un poder inmenso al Estado sobre la cotidianidad de los ciudadanos. Dicha legitimación operaría por analogía. El mercado por su parte se arriesga a ser vivido como un entorno donde se manipula a los individuos, en vez de ser un escenario de decisiones libres, autónomas.

También constituyen dichas tecnologías un ejemplo de la razón instrumental por la nula importancia concedida a la subjetividad de los posibles clientes, y a las eventuales vicisitudes de éstos. Al ser lo dicho un tópico aparte de la eficiencia del sistema para lograr los cometidos internos, resulta obviado. También puede señalarse la posibilidad por parte del propietario de utilizar la casa inteligente para dominar a los demás habitantes de la residencia, lo cual es parte del tipo de racionalidad estudiada, o sea el dominio de la naturaleza y de los sujetos.

La domótica puede operar de modo racional instrumental porque permite el acceso a las pautas de interacción privadas, lo cual podría decantar en un estudio de patrones conductuales. A su vez dicha información muestra la posibilidad de prever conductas y manipularlas, por ejemplo, comercialmente. Tal aspecto es posible dado el paso de datos privados a lo público, esto a su vez se agrava debido al uso de internet, un medio permeable.

El difuminado del sujeto humano se comprueba como consecuencia de las smarthomes, las cuales a la postre son un caso más de la razón instrumental. Los individuos pasan a ser entes previsibles, moldeables en el engranaje de la sociedad industrial. Así, los deseos, los intereses y las necesidades no lo serán más del individuo, sino del mundo administrado en el cual se ubica. Luego de ceder libertad y autonomía al aparato científico-tecnológico, poco quedará de las promesas modernas con sustento en esos dos conceptos políticos. Además, el autoritarismo contará con inmensos recursos de control. 
Quizás, tras el debate político que se aproxima en América Latina, se derive un tratamiento convencional. Las casas inteligentes tal vez pasen a ser arbitradas por comités éticos-científicos para dilucidar cuáles deben ser sus alcances y sus límites. También un comité así daría paso a establecer cuándo una familia, dadas sus calidades, no debe tener una residencia domótica. Sin embargo ¿Puede la institucionalidad evitar todas las potenciales consecuencias negativas de las casas inteligentes? ¿Denunciará sus posibilidades de dominación?

\section{Referencias}

Azimi, I., Rahmani, A., Liljeberg, P., y Tenhunen, H. (2017). Internet of things for remote elderly monitoring: a study from user-centered perspective. Journal of Ambient Intelligence and Humanized Computing, 8(2). 273-289. Doi: 10.1007/s12652-016-0387-y

Bernal, P. (2014). Internet privacy rights. Rights to protect autonomy. Cambridge University Press. Bilogrevic, I., Jadliwala, M, Lám, I., Aad, I., Ginzboorg, P., Niemi, V., Laurent Bindschaedler, L., y Hubaux, J. P. (2012). Big Brother Knows Your Friends: On Privacy of Social Communities in Pervasive Networks. En: Kay, J., Lukowicz, P., Tokuda, H., Patrick Olivier, P., y Krüger, A. (Eds.). Pervasive Computing. (pp. 370-387). Doi: 10.1007/978-3-642-31205-2.

Casas Inteligentes Diseño y Construcción (2016). ¿Qué es una casa inteligente? En: http://www.casasinteligentes.cl/pages/construcciondecasas_casainteligente.htm

Chabot, M., Delaware, L., McCarley, S., Little, C., Nye, A., y Anderson, E. (2019). Living In Place: the Impact of Smart Technology. Current Geriatrics Reports, 8(3). 232-238. Doi: 10.1007/s13670-019-00296-4

De Genaro, E. (2017). O debate da Teoria Crítica sobre a tecnología. Ciências Sociais Unisinos, 53(2). 292-299. Doi: 10.4013/csu.2017.53.2.13

D-link (2016). Home is where the smart is. En: http://us.dlink.com/home-solutions/connectedhome/

Fromm, E. (2002). El miedo a la libertad. Paidós. 
Fuchs, C. (2019). M. N. Roy and the Frankfurt School: Socialist Humanism and the Critical Analysis of Communication, Culture, Technology, Fascism and Nationalism. TripleC: Communication, Capitalism \& Critique, 17(2). 249-286.

Galafassi, G. (2004). Razón instrumental, dominación de la naturaleza y modernidad: la teoría crítica de Max Horkheimer y Theodor Adorno. Theomai, (sin volumen.) (6). Páginas no numeradas.

Germain, G. (2019). The technology trap and the new humanism. South African Journal of Philosophy, 38(2). 127-135. Doi: 10.1080/02580136.2019.1634395

GSMA y KRC Research (2016). GSMA: The impact of the internet of things. The connected home. En: http://www.gsma.com/newsroom/wp-content/uploads/15625-Connected-Living-Report.pdf Hargreaves, T., y Wilson, C. (2017). Smart Homes and Their Users. Doi: 10.1007/978-3-319-68018-7 Hinkelammert, (2003). El sujeto y la ley. El retorno del sujeto reprimido. EUNA. Horkheimer, M, y Adorno, T. (2002). The Dialectics of Enlightenment: Philosophical Fragments. Stanford University Press.

Horkheimer, M. (2000). Teoría tradicional y teoría crítica. Paidós.

Horkheimer, M. (1976). Sociedad en transición: estudios de filosofía social. Ediciones Península.

Horkheimer, M. (1970). Sobre el concepto de hombre y otros ensayos. Editorial Sur.

Horkheimer, M. (1969). Crítica de la razón instrumental. Editorial Sur.

Hortal, A. (2002). Ética general de las profesiones. Desclée de Brouwer.

Kant, E. (1963). (García, M., tr.). Fundamentación de una metafísica de las costumbres. EspasaCalpe.

Keydome (2016). Seguridad. En: http://www.keydome.com/seguridad.html

Kreimer, P. (2017). Los estudios sociales de la ciencia y la tecnología: ¿son parte de las ciencias sociales? Teknokultura, 14(1). 143-162. Doi: 10.5209/TEKN.55727

Ley № 41996-MP-MIDEPLAN. Creación de la Unidad Presidencial de Análisis de Datos. Sistema Costarricense de Información Jurídica.

En: http://www.pgrweb.go.cr/scij/Busqueda/Normativa/Normas/nrm texto completo.aspx?par am1=NRTC\&nValor1=1\&nValor2=90591\&nValor3=119449\&strTipM=TC 
Liu, L., Stroulia, E, Nikolaidis, I, Cruz, M., y Rincon, R., (2016). Smarthomes and home health monitoring technologies for older adults: a systematic review. International Journal of Medical Informatics, 91(Julio). 44-59. Doi: 10.1016/j.ijmedinf.2016.04.007

Locke, J. (2005). (Amor, C., y Stafforini, P., trs.). Ensayo sobre el gobierno civil. Universidad Nacional de Quilmes y Prometeo Libros.

López, P. (2000). Espacios de negación. El legado crítico de Adorno y Horkheimer. Editorial Biblioteca Nueva.

Marcuse, H. (1991). El Hombre Unidimensional. Editorial Joaquín Mortiz.

Marques, G., Pitarma, R., Garcia, N., y Pombo, N. (2019). Internet of Things Architectures, Technologies, Applications, Challenges, and Future Directions for Enhanced Living Environments and Healthcare Systems: A Review. Electronics, 8(1081). 1-27. Doi:10.3390/electronics8101081

Maskeliūnas, R., Damaševičius, R., y Segal, S. (2019). A Review of Internet of Things Technologies for Ambient Assisted Living Environments. Future Internet, 11(259). 1-23. Doi:10.3390/fi11120259

McDonald, A. y Reich, A. (Productores) y Garland, A. (Director). (2015). Ex - Machina [Cinta cinematográfica]. Reino Unido: DNA Films, Universal Pictures International, y Film4 Productions.

Méndez, E. (Comunicación personal, 27 de abril del 2020).

Mumford, L. (1992). Técnica y civilización. Alianza.

Nateras, M. (2009) Reseña de "Crítica de la razón instrumental" de Max Horkheimer. Espacios Públicos, 12(24). 237-240.

Naughton, J. (6 de Diciembre de 2015). Should we be worried if our homes are soon smarter than we are? The Guardian. https://www.theguardian.com/commentisfree/2015/dec/06/smarthomes-security-risk-internet-of-things

Peña, C. (2001). Notas sobre las relaciones entre privacidad y autonomía. En: http://repositorio.uchile.cl/handle/2250/126997 
Putnam, H. (2002). El desplome de la dicotomía hecho-valor y otros ensayos. Paidós.

Rantz, M., Skubic, M., Popescu, M., Galambos, C., Koopman, R., Alexander, G., Phillips, L., Musterman, K., Back, J., y Miller, S. (2015). A New Paradigm of TechnologyEnabled 'Vital Signs' for Early Detection of Health Change for Older Adults. Gerontology, 61(3). 281-290.

Real Academia de la Lengua (2020). Interpretar. En: https://dle.rae.es/interpretar?m=form

Ruiz, A. (29 de Febrero de 2020). Allanan la casa presidencial en Costa Rica por caso de presunta violación de datos. France 24 https://www.france24.com/es/20200228-allanamiento-casapresidencial-costa-rica-datos-alvarado

Shimizu, K. (2017). Reflection, the Public, and the Modern Machine: An Investigation of the Fukushima Disaster in Relation to the Concepts of Truth and Morality. Japanese Journal of Political Science, 18(4), 536-551. Doi:10.1017/S1468109917000160

Slijepčević, P. (2019). Anthropocene, capitalocene, machinocene: Illusions of instrumental reason. Philosophy and Society, 30(4). 543-570. Doi: 10.2298/FID1904543S

Smarthome (2016).

Home center 2. Fibaro System. En:

http://www.smarthome.ec/index.php/productos/item/14-home-center-2

Vargas, M. (7 de Julio de 2016). Seguridad: el principal reto para internet de las cosas. La Nación. http://www.nacion.com/tecnologia/informatica/Seguridad-principal-reto-Internet-

Cosas_0_1571442852.html

Zhang, C. (2013). The evolution of rationality and Modernity crisis. Sociological Mind, 3(2). 179-184. Doi: $10.4236 / \mathrm{sm} .2013 .32024$ 